\title{
Pathophysiological consideration of medullary streaks on FLAIR imaging in pediatric moyamoya disease
}

\author{
Hime Suzuki, MD, ${ }^{1}$ Takeshi Mikami, MD,, Tomoyoshi Kuribara, MD, ${ }^{1}$ Kazuhisa Yoshifuji, MD, ${ }^{2}$ \\ Katsuya Komatsu, MD, ${ }^{1}$ Yukinori Akiyama, MD, ${ }^{1}$ Hirofumi Ohnishi, MD, ${ }^{3}$ Kiyohiro Houkin, MD, ${ }^{4}$ and \\ Nobuhiro Mikuni, MD'
}

\begin{abstract}
Departments of ${ }^{1}$ Neurosurgery and ${ }^{3}$ Public Health, Sapporo Medical University; ${ }^{2}$ Department of Neurosurgery, Hokkaido Medical Center for Child Health and Rehabilitation; and ${ }^{4}$ Department of Neurosurgery, Hokkaido University Graduate School of Medicine, Sapporo, Japan
\end{abstract}

\begin{abstract}
OBJECTIVE Medullary streaks detected on fluid-attenuated inversion recovery (FLAIR) imaging have been considered to be reflected ischemic regions in pediatric moyamoya disease. The purpose of this study was to evaluate these medullary streaks both clinically and radiologically and to discuss associated pathophysiological concerns.
\end{abstract}

METHODS The authors retrospectively reviewed data from 14 consecutive pediatric patients with moyamoya disease treated between April 2009 and June 2016. Clinical and radiological features and postoperative imaging changes were analyzed. In 4 patients, hyperintense medullary streaks on FLAIR imaging (HMSF) at the level of the centrum semiovale were detected.

RESULTS The HMSF were coincident with hyperintense medullary streaks on a T2-weighted image, though they were not completely coincident with the vasculature on either a T2*-weighted image or contrast-enhanced CT. Analysis revealed significantly higher values in terms of MR angiography scores, number of flow voids of the basal ganglia, and the presence of the medullary artery in the group with HMSF than in those without. In contrast, the presence of white matter damage was significantly less frequent in the HMSF group. All HMSF disappeared after surgery, and the mean apparent diffusion coefficient at the same level was significantly reduced postoperatively.

CONCLUSIONS Although HMSF should be associated with collateral circulation in moyamoya disease, other factors may be involved, including stagnated cerebrospinal fluid or vasogenic edema that is relevant to the impaired state of the white matter. Findings in this study provide insight into the pathophysiological basis of the perivascular space in moyamoya disease.

https://thejns.org/doi/abs/10.3171/2017.1.PEDS16541

KEY WORDS moyamoya disease; medullary streaks; MRI; vascular disorders

$\mathrm{M}$ OYAMOYa disease is a cerebrovascular disorder characterized by chronic progressive stenotic or occlusive change of the terminal portions of the bilateral internal carotid arteries (ICAs), which induces an abnormal vascular network composed of collateral pathways at the base of the brain. ${ }^{25,33}$ Ischemic and hemorrhagic events are caused by the stenotic or occlusive change of these arteries and vulnerable collateral vessels. Although angiography has been the principal method used in diagnosis, novel characteristic findings of moyamoya disease have been depicted through recent advances in 3-T MRI technology. 11,18,23,29 These developments have provided the opportunity to consider the pathophysiology of moyamoya disease. Fast imaging employing steady-state acquisition demonstrates regression of the middle cerebral artery (MCA), which suggests the remodeling pattern of the major vessels. ${ }^{22}$ Flow-sensitive black-blood MR angiography (MRA) signals from rapidly flowing blood can visualize small perforating arteries, thus detailing morphological changes in collateral vessels in moyamoya dis-

ABBREVIATIONS ACA = anterior cerebral artery; $A D C=$ apparent diffusion coefficient; $C S F=$ cerebrospinal fluid; $C T A=C T$ angiography; $D W I=$ diffusion-weighted imaging; FLAIR = fluid-attenuated inversion recovery; HMSF = hypointense medullary streaks on FLAIR imaging; ICA = internal carotid artery; IMSF = isointense medullary streaks on FLAIR imaging; MCA = middle cerebral artery; MRA = MR angiography; PCA = posterior cerebral artery; $R O I=$ region of interest; STA = superficial temporal artery.

SUBMITTED September 23, 2016. ACCEPTED January 11, 2017.

INCLUDE WHEN CITING Published online March 10, 2017; DOI: 10.3171/2017.1.PEDS16541. 
ease. ${ }^{5,22}$ The progression of periventricular anastomosis reflects the hemorrhagic events because periventricular anastomosis is histologically fragile, is possibly subject to long-standing hemodynamic stress, and is associated with microaneurysms, $5,6,37$ and detecting these anastomoses is extremely important. In terms of deep white matter change, increased white matter lesions have been indicated in moyamoya disease, ${ }^{17}$ and the elevation in the apparent diffusion coefficient (ADC) in normal-appearing white matter in moyamoya disease is associated with cerebrovascular reactivity impairment. ${ }^{4,13}$

Linear hyperintensity along the sulci on fluid-attenuated inversion recovery (FLAIR) imaging is referred to as the "leptomeningeal ivy sign," is considered to indicate retrograde slow flow of the leptomeningeal anastomosis produced by the stenotic or occlusive change in the major vessels, ${ }^{20}$ and is indicative of misery perfusion, which is hemodynamic compromise due to impaired cerebral blood flow. ${ }^{15,24,36}$ In some pediatric cases, the linear hyperintensity on FLAIR imaging extends to the perivascular space of the deep white matter, ${ }^{16,20,31,38}$ is referred to as "medullary streaks," and is considered to be associated with changes in vasculature. ${ }^{34}$ The aim of this study was to investigate the characteristics of medullary streaks on FLAIR imaging in pediatric moyamoya disease and to explore the pathophysiology of the findings.

\section{Methods}

\section{Patients}

Between April 2009 and June 2016, 14 consecutive pediatric patients with moyamoya disease were treated at Sapporo Medical University. All of the patients had been diagnosed with moyamoya disease via MRI, MRA, and digital subtraction angiography, according to diagnostic guidelines. ${ }^{29}$ Data from these 14 patients were retrospectively analyzed in this study. One of these cases represented unilateral involvement. The mean patient age was $8.2 \pm 4.0$ years (range 2-14 years). Seven patients were male and 7 were female. Initial clinical symptoms in the patients were as follows: transient ischemic attack (9 patients), ischemic stroke (2 patients), headache (1 patient), involuntary movement ( 1 patient), and none ( 1 patient). In 8 hemispheres in 4 patients, medullary streaks exhibited hyperintensity on FLAIR sequences (Fig. 1). The evaluated T2-weighted and FLAIR images were merged on a workstation to judge whether the demonstrated hyperintense medullary streaks coincided with one another. Similarly, the FLAIR image was merged with the T2*-weighted image and contrast-enhanced CT scan to judge whether the hyperintense medullary streaks represented the medullary arteries and veins. Next, the 28 brain hemispheres of the 14 patients were divided into 2 groups, according to the signal intensity (hyperintense or isointense) of the medullary streaks on FLAIR imaging. Patients with hyperintense medullary streaks on FLAIR imaging (HMSF) were compared to patients with isointense medullary streaks on FLAIR imaging (IMSF) with regard to the following clinical and radiological features: age, sex, initial clinical symptom, MRA score, number of flow voids of the basal ganglia, development of periventricular anastomosis, presence of

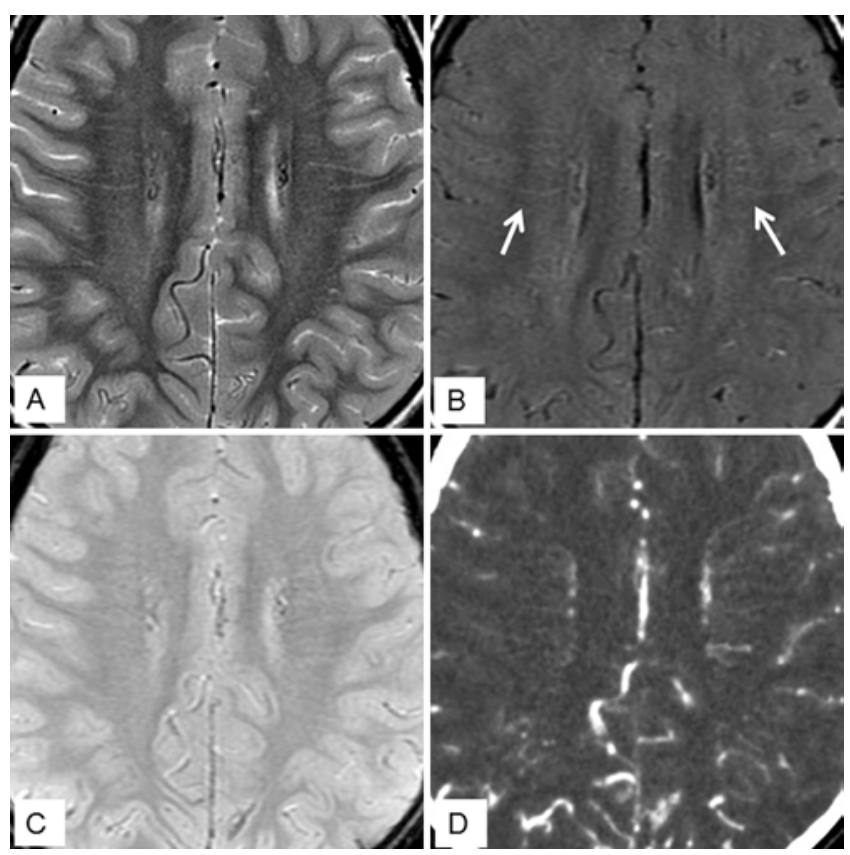

FIG. 1. Axial T2-weighted MR image (A) showing medullary streaks at the level of the centrum semiovale. Axial FLAIR image (B) showing hyperintense medullary streaks (arrows). Axial T2*-weighted image (C) demonstrating vascular hyposignal. Axial contrast-enhanced CT (D) showing vascular enhancement.

white matter damage (large $[>1 \mathrm{~cm}]$ stroke lesions, diffuse hemispheric brain atrophy, and cystic periventricular leukomalacia), and extent of leptomeningeal ivy sign. Two independent observers (H.S. and T.K.) performed the imaging review to determine HMSF or IMSF while blinded to the other clinical and radiological factors.

Surgery was performed in 19 hemispheres in 10 patients. The operative method was superficial temporal artery (STA) to MCA bypass and encephaloduromyosynangiosis in all 19 hemispheres. Among the patients, the 4 with HMSF participated in a follow-up study at 3 months and again at 1 year after surgery. In the 8 hemispheres with HMSF, the postoperative change in signal intensity on FLAIR imaging was evaluated. The ADC at the level of the centrum semiovale on diffusion-weighted imaging (DWI) was also assessed for white matter integrity. The imaging reviewers (H.S. and T.K.), again blinded to the other clinical and radiological factors, scored the pre- and postoperative ADC, and the mean value was assessed.

\section{MRI and MRA Examination}

Magnetic resonance imaging examination was performed using a 3-T system (Signa Excite, version 11, GE Medical Systems). The MRA was performed using the 3D time-of-flight technique with a 3D spoiled gradientrecalled echo (3D TOF SPGR) sequence. The details on imaging parameters for the T2-weighted, FLAIR, T2*weighted, and 3D TOF SPGR sequences at our institute have been mentioned elsewhere. ${ }^{17,26}$ The relationship between the HMSF and an extensive ivy sign was evaluated using the ivy sign scoring adapted from a previous report. ${ }^{24}$ The region of the anterior cerebral artery (ACA), 
anterior half of the MCA region (ant-MCA), posterior half of the MCA region (post-MCA), and the region of the posterior cerebral artery (PCA) were involved in this analysis. The ant-MCA and post-MCA regions were separated by the central sulcus. The extent of the ivy sign (ivy sign score) in each region was scored ( $0-2$ points): 0 points indicated the absence of an ivy sign, 1 point indicated an ivy sign on less than half of the cortical surface in each region, and 2 points indicated an ivy sign on more than half of the cortical surface. The total points for each region per hemisphere were calculated and then divided into 2 groups: extensive ivy sign $(\geq 3)$ and focal ivy sign $(\leq 2)$.

Magnetic resonance angiography scoring and the score for each of the vessels were based on Houkin et al.'s classification. ${ }^{12,29}$ Depending on the severity of the stenotic or occlusive change, an MRA score was assigned to each of the following: $\mathrm{C}_{1}$ portion of the ICA, $\mathrm{M}_{2}$ portion of the MCA, $\mathrm{A}_{1}$ portion of the ACA, and $\mathrm{P}_{2}$ portion of the PCA. The minimum possible score was 0 and the maximum was $10(\mathrm{ICA} 3$ + MCA3 + ACA2 + PCA2 = 10). For each patient, the total score was calculated. The number of flow voids of the basal ganglia was obtained on a 3D TOF SPGR imaging slice of the basal ganglia. The stroke lesion size, the presence of hemispheric brain atrophy, and the presence of cystic periventricular leukomalacia were evaluated on T2-weighted imaging. The leptomeningeal ivy sign was judged from FLAIR imaging. Diffusion-weighted imaging was performed using a single-shot echo-planar sequence with the following protocol: repetition time 5000 msec; echo time 80.9 msec; FOV $200 \times 200 \mathrm{~mm}$; matrix $128 \times 192$; slice thickness $4 \mathrm{~mm}$; slice gap $1 \mathrm{~mm}$; number of excitations 1 ; and $\mathrm{b}=0$ and 1500 seconds $/ \mathrm{mm}^{2}$ with 3 principal axes. The resulting DWI and ADC data were transferred to the workstation for analysis. Three regions of interest (ROIs) were placed on the ADC map at the level of the centrum semiovale, and the mean value of each hemisphere was calculated. The shape of each ROI was a square with sides of $1 \mathrm{~cm}$.

\section{Assessment of the Collateral Vessels}

Of the periventricular anastomoses, the presence of the medullary artery that passed through the centrum semiovale was assessed using 3D CT angiography (CTA). The threshold of the opacity curve was set to half of the CT value in the basilar artery. Computed tomography angiog- raphy examination was performed using a multidetector row CT (Aquilion ONE, Toshiba Medical Systems Corp.). The 3D CTA scanning parameters, protocol, and postprocessing technique have been previously mentioned. ${ }^{32}$ The information was transferred to a workstation (Ziostation 2, Ziosoft Inc.), and 3D multicolored images were produced. A coronal image of thick-slab maximum intensity projection at the level of the thalamus was applied as a postprocessing technique to aid evaluation. The presence of the medullary artery was judged on the image in the hemisphere with at least 3 enhanced vessels.

\section{Statistical Analysis}

Data are expressed as the means \pm standard deviations. The Mann-Whitney U-test and Fisher's exact probability tests were used for comparing the HMSF and IMSF cases. The Wilcoxon signed-rank test was used to compare preoperative and postoperative ADC values. All statistical analyses were conducted using an SPSS software package (version 24, IBM Corp.), and $\mathrm{p}<0.05$ was considered to be indicative of statistical significance.

\section{Results}

In 4 cases, the HMSF was merged with the T2-weighted image, T2*-weighted image, and contrast-enhanced CT. On the merged image, the HMSF coincided with the hyperintense medullary streaks on the T2-weighted image (Fig. 2), though they were not coincident with the vascular hyposignal on the T2*-weighted image, nor with the vascular enhancement on contrast-enhanced CT.

The clinical and radiological characteristics of the patients are presented in Table 1. The mean patient age in the HMSF group was 7.3 \pm 2.2 years (range 5-10 years), while that in the IMSF group was $8.6 \pm 4.6$ years (range 2-14 years). Although the HMSF patients were somewhat younger than those in the IMSF group, the difference did not reach statistical significance $(p=0.733)$. There were no significant differences between these 2 groups in terms of sex and initial clinical symptoms. The MRA score was significantly higher for the HMSF group $(6.9 \pm 1.1)$ than for the IMSF group $(3.9 \pm 1.8, \mathrm{p}<0.001)$. The number of flow voids of the basal ganglia was significantly higher for the HMSF group $(14.4 \pm 6.2)$ than for the IMSF group $(3.6 \pm 3.0, \mathrm{p}<0.001)$. The medullary artery was visible on

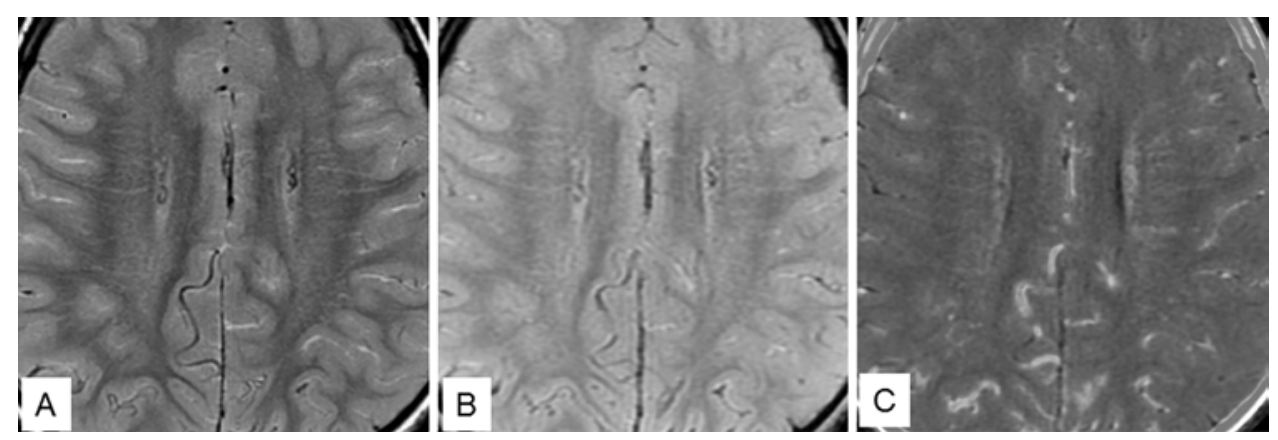

FIG. 2. A merged T2-weighted and FLAIR image (A). The HMSF was coincident with the hyperintensity on the T2-weighted image. However, it was not coincident with the vascular hyposignal of a $\mathrm{T}^{*}$-weighted image (B), nor with the vascular enhancement on a merged FLAIR image and CT angiogram (C). 
TABLE 1. Clinical characteristics of 14 patients with moyamoya disease

\begin{tabular}{|c|c|c|c|}
\hline Parameter & HMSF Group & IMSF Group & p Value \\
\hline No. of patients & 4 & 10 & \\
\hline No. of hemispheres & 8 & 20 & \\
\hline Age in yrs (range) & $7.3 \pm 2.2(5-10)$ & $8.6 \pm 4.6(2-14)$ & 0.733 \\
\hline $\operatorname{Sex}(M / F)$ & $1 / 3$ & $6 / 4$ & 0.254 \\
\hline \multicolumn{4}{|l|}{ Initial clinical symptom } \\
\hline TIA & 4 & 5 & \multirow{3}{*}{0.217} \\
\hline Ischemic stroke & 0 & 2 & \\
\hline Other & 0 & 3 & \\
\hline MRA score & $6.9 \pm 1.1$ & $3.9 \pm 1.8$ & $<0.001^{*}$ \\
\hline $\begin{array}{l}\text { No. of flow voids of } \\
\text { basal ganglia }\end{array}$ & $14.4 \pm 6.2$ & $3.6 \pm 3.0$ & $<0.001^{*}$ \\
\hline Medullary artery on CTA & $8 / 8$ & $3 / 20$ & $<0.001^{*}$ \\
\hline $\begin{array}{l}\text { Diffuse white matter } \\
\text { damage }\end{array}$ & $0 / 8$ & $8 / 20$ & $0.041^{*}$ \\
\hline $\begin{array}{l}\text { Stroke lesion (>1 } \\
\quad \mathrm{cm})\end{array}$ & 0 & 2 & \\
\hline $\begin{array}{l}\text { Diffuse hemispher- } \\
\text { ic brain atrophy }\end{array}$ & 0 & 5 & \\
\hline $\begin{array}{l}\text { Cystic periventricu- } \\
\text { lar leukomalacia }\end{array}$ & 0 & 4 & \\
\hline $\begin{array}{l}\text { Ivy sign score (extensive } \\
\text { group/focal group) }\end{array}$ & $4 / 4$ & $3 / 17$ & 0.142 \\
\hline
\end{tabular}

$\mathrm{TIA}=$ transient ischemic attack.

* Indicates statistical significance.

CTA significantly more often in the HMSF group than in the IMSF group $(\mathrm{p}<0.001)$. As regards white matter damage, a large stroke lesion $(>1 \mathrm{~cm})$ was found in 2 hemispheres, diffuse hemispheric brain atrophy was found in 5 hemispheres, and cystic periventricular leukomalacia was found in 4 hemispheres. Of these, multiple lesions of white matter damage were found in 3 hemispheres. The presence of diffuse white matter damage was significantly less frequent in the HMSF group than in the IMSF group ( $p$ $=0.041$ ). Eight hemispheres in the IMSF group presented with white matter damage; nevertheless, these revealed a developed medullary artery on CTA. The presence of the leptomeningeal ivy sign was confirmed in all hemispheres in the HMSF group and in 16 hemispheres $(80.0 \%)$ in the IMSF group. Four hemispheric sides $(50.0 \%)$ in the HMSF group and 3 hemispheric sides $(15.0 \%)$ in the IMSF group were included in the extensive group. There was no significant difference between these 2 groups $(p=0.142$ ).

Among the 4 patients (8 surgical procedures) who had the HMSF and participated in the postoperative follow-up study, there were no instances of perioperative cerebral infarction or postoperative hyperperfusion syndrome. In all patients who experienced transient ischemic attacks, these attacks disappeared during the follow-up period. Patency of the STA-MCA bypass and the development of indirect anastomosis was confirmed on MRA in all patients during follow-up. Postoperative changes in HMSF are represented in Fig. 3. The HMSF recognized preoperatively (Fig. 3A and B) was decreased only on the operative side

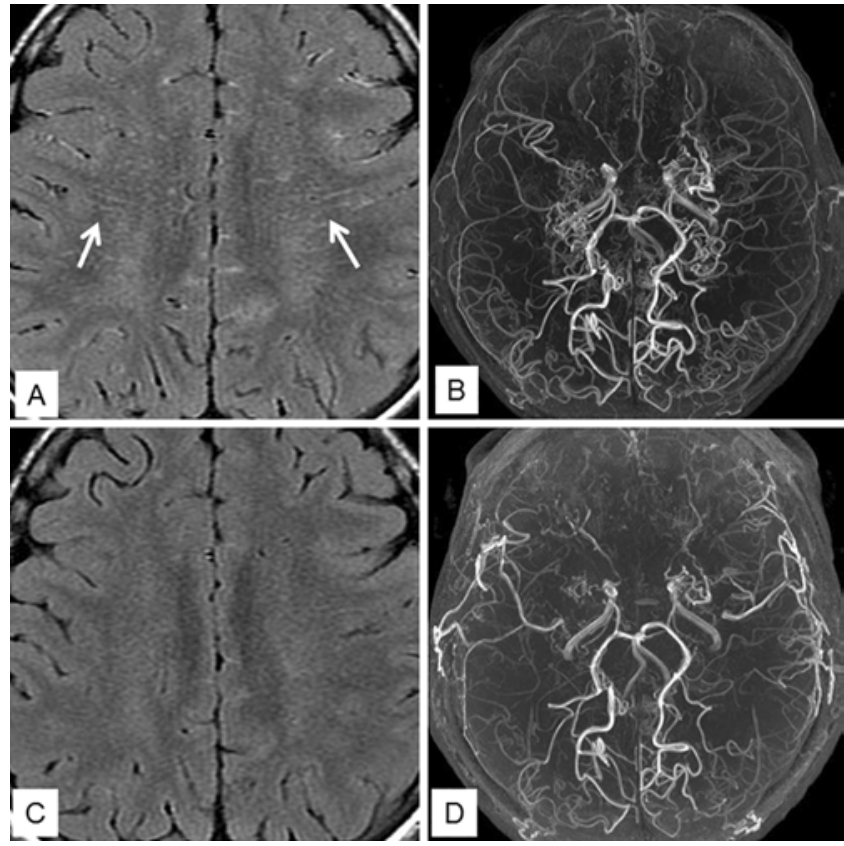

FIG. 3. Preoperative FLAIR image (A) and MRA study (B) obtained in an 8-year-old female. Arrows indicate HMSF. Twelve-month postoperative FLAIR image (C) and MRA study (D). The HMSF disappeared bilaterally after bilateral surgery, and collateral flow from the STA, deep temporal artery, and middle meningeal artery developed.

12 months after revascularization surgery on the left side (Fig. 3C and D). The HMSF disappeared bilaterally after bilateral revascularization surgery. Signal decrease in the HMSF was confirmed in all 8 hemispheres in the 4 patients after revascularization surgery. Figure 4 shows the change in ADC of the centrum semiovale in the HMSF cases after revascularization surgery. The mean ADC measured preoperatively was $734.2 \pm 63.9$ (range 626.5851.8 ), whereas postoperatively it was $702.0 \pm 60.4$ (range 596.2-796.0). The postoperative ADC decreased 4.4\% compared with preoperative ADC, which was statistically significantly different $(p=0.001)$.

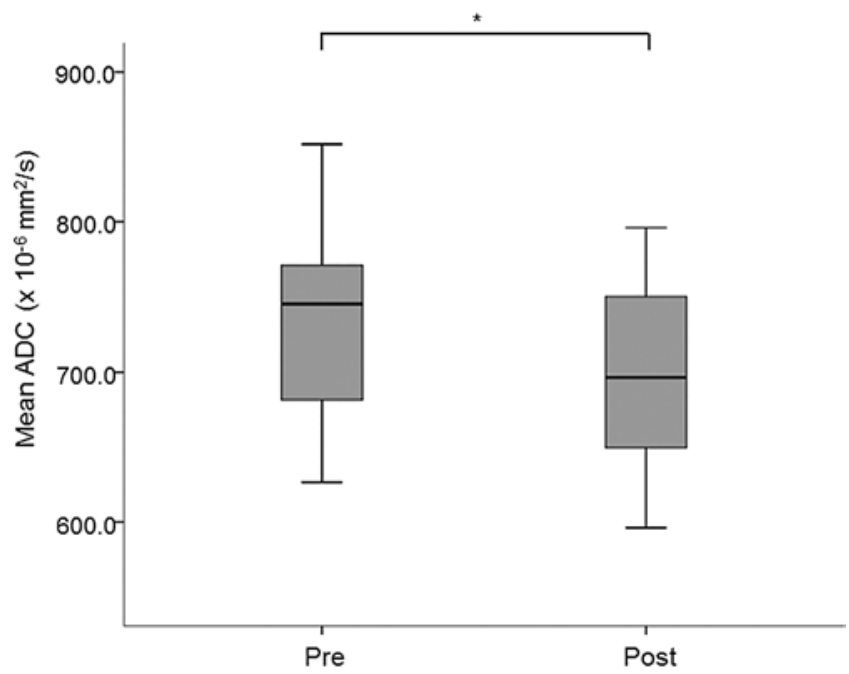

FIG. 4. The mean ADC was significantly decreased postoperatively ( $p$ $=0.001$ ). 


\section{Discussion Medullary Streaks}

Medullary streaks were first described in 2001 by Harada et al. ${ }^{11}$ as "linear structures crossing the white matter of the brain." In patients with moyamoya disease, medullary streaks may be associated with decreased cerebral blood flow, ${ }^{11}$ and they have been recognized in adults with atherosclerotic disease. ${ }^{21}$ Basically, medullary streaks were observed as hypersignals on T2-weighted imaging, though they were recognized as hypersignals on FLAIR imaging in some pediatric patients with moyamoya disease. ${ }^{34}$ In previous reports, these HMSF were considered an extension of the ivy sign. . $^{16,20,31,38}$

\section{Pathophysiology of HMSF}

Given our results, we suggest that the HMSF could be caused by at least 3 possible factors, the first of which is associated with vasculature. The number of flow voids of the basal ganglia and the presence of the medullary artery were significantly higher in the HMSF cases in our study. Takanashi et al. speculated, based on the findings of postoperative normalization, that medullary streaks are related to ischemia, which is attributable to the dilation of medullary arteries acting as collateral flow. ${ }^{34}$ Harada et al. concurred, as their data showed a postoperative decrease in the medullary streak diameter. ${ }^{11}$ The paraventricular anastomosis should be associated either directly or indirectly with the appearance of the HMSF. However, some of our findings contradicted those of Takanashi and Harada. The HMSF was not coincident with the vasculature of medullary arteries on the merged CTA image, and it was not consistent with the leptomeningeal ivy sign. Additionally, it was not found in cases with white matter damage; nevertheless, these cases exhibited developed medullary arteries. Moreover, the HMSF have been reported only in pediatric cases s $^{16,20,31,38}$ and not in adult cases, even those involving developed collateral vasculature. Consequently, the vasculature is not a direct cause of HMSF. The appearance of HMSF may represent the reversible ischemic impairment of the white matter before a damaged state.
On this basis, detection and management of HMSF should be considered clinically important for achieving prognostic control.

The second possibility is that HMSF represents cerebrospinal fluid (CSF) stagnation in the perivascular space. The relatively high white matter volume and intracranial pressure present from the ages of roughly 6-12 years may accelerate CSF retention in the perivascular space. The perivascular space consists of gaps containing CSF, and it surrounds small blood vessels penetrating the brain parenchyma. ${ }^{19}$ An enlarged perivascular space has appeared more often in the basal ganglia and the centrum semiovale encompassing the lenticulostriate artery., ${ }^{2,3}$ The CSF of the perivascular space usually circulates by para-arterial influx due to arterial pulsation or respiration, and convective flow facilitates the clearance of interstitial solutes from the brain (Fig. 5A). An enlarged perivascular space is associated with aging, atherosclerosis, and hypertension due to the reduction in the expansion property of arterioles. ${ }^{10,30}$ The dysfunction of vascular endothelial cells then promotes the leakage of plasma proteins to perivascular compartments through the reduction of para-arterial influx (Fig. 5B). Consequently, the small vessels' disease represents an enlarged perivascular space, white matter lesions, lacunar stroke, and microbleeds. ${ }^{28}$ An enlarged perivascular space in moyamoya disease could be similarly explained by the reduced pulsation of arterial flow via ICA stenotic or occlusive changes that induce a dysfunction of perivascular clearance. There is evidence that lymphocytes traffic from the brain with CSF via the cribriform plate and nasal mucosa to cervical lymph nodes. ${ }^{9}$ Recent findings concerning the perivascular space have suggested that the perivascular space-mediated CSF flow be called "the glymphatic system" and that it leads to the pathophysiological clarification of cerebrovascular disorders as well as Alzheimer's disease and normal-pressure hydrocephalus., ${ }^{714}$ An enlarged perivascular space in moyamoya disease could also be explained by destruction of the system, and we think that this theory is most supported by our findings in the present study.

On the other hand, perhaps the HMSF indicate an
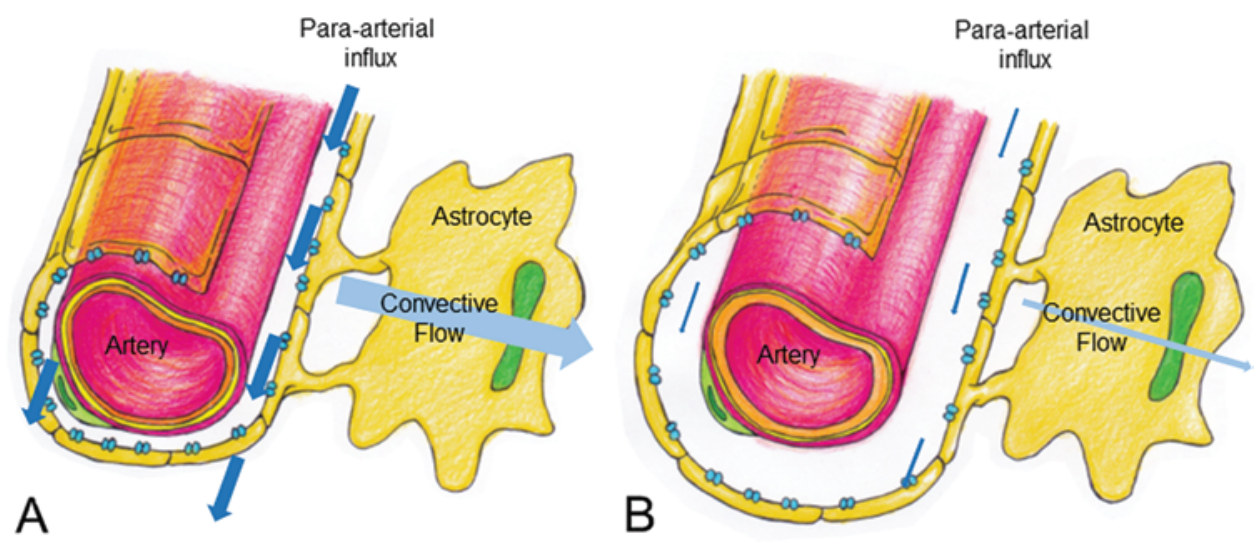

FIG. 5. Illustrated models of the perivascular space in healthy and moyamoya disease cases. In healthy individuals (A), CSF enters the brain parenchyma via para-arterial influx, and convective flow facilitates the clearance of interstitial solutes from the brain. In moyamoya disease cases (B), a reduction in arterial flow leads to abnormal enlargement of the perivascular space through the reduction of para-arterial influx and convective flow. Copyright Takeshi Mikami. Published with permission. Figure is available in color online only. 
edematous change around the perivascular space. In cases involving HMSF, the ADC value was significantly reduced postoperatively. An increase in the ADC in normal-appearing white matter in cases involving ischemia has been thought to be associated with increased water diffusivity due to Wallerian degeneration of axons. ${ }^{27}$ The improvement in vasogenic edema around the perivascular space should also be considered. In the evolution of multiple sclerosis lesions, perivascular space inflammation is a critical event in the pathogenesis. ${ }^{8}$ This has been confirmed by histopathological study of multiple sclerosis, which shows that intense perivenular cuffing by lymphocytes and monocytes in lesion plaques is found in active acute lesions. ${ }^{1,35}$ Perivascular space inflammation is considered to be associated with the disruption of the bloodbrain barrier, myelin breakdown, and the formation of new lesions. In moyamoya disease, the perivascular space has not been examined histopathologically, and further study is expected in the future.

\section{Study Limitations}

There are some limitations to this study. First, it involved a relatively small number of participants and only 4 cases of HMSF. Therefore, the statistical analysis was performed using a nonparametric test for all. Moyamoya disease is rare, and HMSF were only detected in pediatric cases. Therefore, we could not perform a multivariate analysis. However, HMSF cases shared some characteristics, and our preliminary data should prove essential. Further clinical and radiological analysis with a larger number of cases may verify our findings. Second, since the properties of moyamoya disease vary in each hemisphere, our statistical analysis involved both hemispheres. Although the same tendency was found in the calculation per patient, that did not reflect the radiological characteristics of the hemispheres. Thus, the results of our study were calculated per hemisphere. Third, the HMSF indicate an extremely small lesion, and our conjecture could not be completely proven with any MRI sequence. In future work, an appropriate modality with higher spatial resolution, contrast resolution, and time resolution will be needed for a more detailed analysis. Additionally, a pathological analysis may be necessary.

\section{Conclusions}

We discussed the pathophysiological basis of medullary streaks in pediatric moyamoya disease. Although the HMSF were associated with collateral circulation, they were not completely coincident with the vasculature. Given our results, it is possible that the HMSF are related not only through the collateral flow of paraventricular anastomosis, but also through the stagnated CSF in or the vasogenic edema around the perivascular space. At any rate, the appearance of HMSF may represent reversible white matter impairment before a damaged state, and detection and management of HMSF should be considered clinically important. We think that our results provide insight into the pathophysiological basis of the perivascular space in moyamoya disease.

\section{References}

1. Adams CW, Poston RN, Buk SJ, Sidhu YS, Vipond H: Inflammatory vasculitis in multiple sclerosis. J Neurol Sci 69:269-283, 1985

2. Bokura H, Kobayashi S, Yamaguchi S: Distinguishing silent lacunar infarction from enlarged Virchow-Robin spaces: a magnetic resonance imaging and pathological study. J Neurol 245:116-122, 1998

3. Braffman BH, Zimmerman RA, Trojanowski JQ, Gonatas NK, Hickey WF, Schlaepfer WW: Brain MR: pathologic correlation with gross and histopathology. 1. Lacunar infarction and Virchow-Robin spaces. AJR Am J Roentgenol 151:551-558, 1988

4. Calviere L, Ssi Yan Kai G, Catalaa I, Marlats F, Bonneville F, Larrue V: Executive dysfunction in adults with moyamoya disease is associated with increased diffusion in frontal white matter. J Neurol Neurosurg Psychiatry 83:591-593, 2012

5. Funaki T, Fushimi Y, Takahashi JC, Takagi Y, Araki Y, Yoshida K, et al: Visualization of periventricular collaterals in moyamoya disease with flow-sensitive black-blood magnetic resonance angiography: preliminary experience. Neurol Med Chir (Tokyo) 55:204-209, 2015

6. Funaki T, Takahashi JC, Yoshida K, Takagi Y, Fushimi Y, Kikuchi T, et al: Periventricular anastomosis in moyamoya disease: detecting fragile collateral vessels with MR angiography. J Neurosurg 124:1766-1772, 2016

7. Gaberel T, Gakuba C, Goulay R, Martinez De Lizarrondo S, Hanouz JL, Emery E, et al: Impaired glymphatic perfusion after strokes revealed by contrast-enhanced MRI: a new target for fibrinolysis? Stroke 45:3092-3096, 2014

8. Ge Y, Law M, Herbert J, Grossman RI: Prominent perivenular spaces in multiple sclerosis as a sign of perivascular inflammation in primary demyelination. AJNR Am J Neuroradiol 26:2316-2319, 2005

9. Goldmann J, Kwidzinski E, Brandt C, Mahlo J, Richter D, Bechmann I: T cells traffic from brain to cervical lymph nodes via the cribroid plate and the nasal mucosa. J Leukoc Biol 80:797-801, 2006

10. Gutierrez J, Rundek T, Ekind MS, Sacco RL, Wright CB: Perivascular spaces are associated with atherosclerosis: an insight from the Northern Manhattan Study. AJNR Am J Neuroradiol 34:1711-1716, 2013

11. Harada A, Fujii Y, Yoneoka Y, Takeuchi S, Tanaka R, Nakada T: High-field magnetic resonance imaging in patients with moyamoya disease. J Neurosurg 94:233-237, 2001

12. Houkin K, Nakayama N, Kuroda S, Nonaka T, Shonai T, Yoshimoto T: Novel magnetic resonance angiography stage grading for moyamoya disease. Cerebrovasc Dis 20:347354,2005

13. Jeong H, Kim J, Choi HS, Kim ES, Kim DS, Shim KW, et al: Changes in integrity of normal-appearing white matter in patients with moyamoya disease: a diffusion tensor imaging study. AJNR Am J Neuroradiol 32:1893-1898, 2011

14. Jessen NA, Munk AS, Lundgaard I, Nedergaard M: The glymphatic system: a beginner's guide. Neurochem Res 40:2583-2599, 2015

15. Kaku Y, Iihara K, Nakajima N, Kataoka H, Fukushima K, Iida $\mathrm{H}$, et al: The leptomeningeal ivy sign on fluid-attenuated inversion recovery images in moyamoya disease: positron emission tomography study. Cerebrovasc Dis 36:19-25, 2013

16. Kawashima M, Noguchi T, Takase Y, Nakahara Y, Matsushima T: Decrease in leptomeningeal ivy sign on fluid-attenuated inversion recovery images after cerebral revascularization in patients with moyamoya disease. AJNR Am J Neuroradiol 31:1713-1718, 2010

17. Komatsu K, Mikami T, Noshiro S, Miyata K, Wanibuchi M, Mikuni N: Reversibility of white matter hyperintensity by revascularization surgery in moyamoya disease. J Stroke Cerebrovasc Dis 25:1495-1502, 2016 
18. Kuroda S, Houkin K: Moyamoya disease: current concepts and future perspectives. Lancet Neurol 7:1056-1066, 2008

19. Kwee RM, Kwee TC: Virchow-Robin spaces at MR imaging. Radiographics 27:1071-1086, 2007

20. Maeda M, Tsuchida C: "Ivy sign" on fluid-attenuated inversion-recovery images in childhood moyamoya disease. AJNR Am J Neuroradiol 20:1836-1838, 1999

21. Mikami C, Inoue T, Ogasawara K, Ogawa A: Medullary streaks in a patient with atherosclerotic internal carotid artery occlusion: case report. Surg Neurol 62:42-44, 2004

22. Mikami T, Noshiro S, Komatsu K, Miyata K, Akiyama Y, Wanibuchi M, et al: Vascular remodeling of the circle of Willis in moyamoya disease. Neurol Res 37:880-885, 2015

23. Mikami T, Sugino T, Ohtaki S, Houkin K, Mikuni N: Diagnosis of moyamoya disease on magnetic resonance imaging: are flow voids in the basal ganglia an essential criterion for definitive diagnosis? J Stroke Cerebrovasc Dis 22:862-868, 2013

24. Mori N, Mugikura S, Higano S, Kaneta T, Fujimura M, Umetsu A, et al: The leptomeningeal "ivy sign" on fluidattenuated inversion recovery MR imaging in moyamoya disease: a sign of decreased cerebral vascular reserve? AJNR Am J Neuroradiol 30:930-935, 2009

25. Nishimoto A, Takeuchi S: Abnormal cerebrovascular network related to the internal carotid arteries. J Neurosurg 29:255-260, 1968

26. Noshiro S, Mikami T, Komatsu K, Miyata K, Akiyama Y, Wanibuchi M, et al: Cortical and subcortical vascular hypointensity on $\mathrm{T} 2 *$ weighted imaging in moyamoya disease. Neurol Res 38:110-116, 2016

27. O'Sullivan M, Summers PE, Jones DK, Jarosz JM, Williams SC, Markus HS: Normal-appearing white matter in ischemic leukoaraiosis: a diffusion tensor MRI study. Neurology 57:2307-2310, 2001

28. Pantoni L: Cerebral small vessel disease: from pathogenesis and clinical characteristics to therapeutic challenges. Lancet Neurol 9:689-701, 2010

29. Research Committee on the Pathology and Treatment of Spontaneous Occlusion of the Circle of Willis: Guidelines for diagnosis and treatment of moyamoya disease (spontaneous occlusion of the circle of Willis). Neurol Med Chir (Tokyo) 52:245-266, 2012

30. Rouhl RP, van Oostenbrugge RJ, Knottnerus IL, Staals JE, Lodder J: Virchow-Robin spaces relate to cerebral small vessel disease severity. J Neurol 255:692-696, 2008

31. Seo KD, Suh SH, Kim YB, Kim JH, Ahn SJ, Kim DS, et al: Ivy sign on fluid-attenuated inversion recovery images in moyamoya disease: correlation with clinical severity and old brain lesions. Yonsei Med J 56:1322-1327, 2015
32. Suzuki H, Mikami T, Komatsu K, Noshiro S, Miyata K, Hirano T, et al: Assessment of the cortical artery using computed tomography angiography for bypass surgery in moyamoya disease. Neurosurg Rev [epub ahead of print], 2016

33. Suzuki J, Takaku A: Cerebrovascular "moyamoya" disease. Disease showing abnormal net-like vessels in base of brain. Arch Neurol 20:288-299, 1969

34. Takanashi J, Suzuki H, Barkovich AJ, Sugita K, Saeki N, Kobayashi E, et al: Medullary streaks: dilated medullary vessels in chronic ischemia in children. Neurology 61:583-584, 2003

35. Tanaka R, Iwasaki Y, Koprowski H: Ultrastructural studies of perivascular cuffing cells in multiple sclerosis brain. Am J Pathol 81:467-478, 1975

36. Vuignier S, Ito M, Kurisu K, Kazumata K, Nakayama N, Shichinohe H, et al: Ivy sign, misery perfusion, and asymptomatic moyamoya disease: FLAIR imaging and ${ }^{15} \mathrm{O}$-gas positron emission tomography. Acta Neurochir (Wien) 155:2097-2104, 2013

37. Yamashita M, Oka K, Tanaka K: Histopathology of the brain vascular network in moyamoya disease. Stroke 14:50-58, 1983

38. Yoon HK, Shin HJ, Chang YW: "Ivy sign" in childhood moyamoya disease: depiction on FLAIR and contrast-enhanced T1-weighted MR images. Radiology 223:384-389, 2002

\section{Disclosures}

The authors report no conflict of interest concerning the materials or methods using in this study or the findings specified in this paper.

\section{Author Contributions}

Conception and design: Mikami. Acquisition of data: Suzuki, Kuribara, Komatsu. Analysis and interpretation of data: Mikami, Suzuki, Kuribara, Komatsu, Ohnishi. Drafting the article: Suzuki. Critically revising the article: Mikami, Suzuki. Reviewed submitted version of manuscript: Mikami, Kuribara, Yoshifuji, Ohnishi. Approved the final version of the manuscript on behalf of all authors: Mikami. Statistical analysis: Mikami, Ohnishi. Administrative/technical/material support: Kuribara, Yoshifuji, Komatsu, Akiyama, Houkin, Mikuni. Study supervision: Mikami, Yoshifuji, Akiyama, Houkin, Mikuni.

\section{Correspondence}

Takeshi Mikami, Department of Neurosurgery, Sapporo Medical University, South 1 West 16, Chuo-ku, Sapporo 060-8543, Japan. email: tmikami@sapmed.ac.jp. 